\title{
Klein Topological Field Theories from Group Representations
}

\author{
Sergey A. LOKTEV ${ }^{\dagger \ddagger}$ and Sergey M. NATANZON ${ }^{\dagger \ddagger \S}$ \\ $\dagger$ Department of Mathematics, Higher School of Economics, \\ 7 Vavilova Str., Moscow 117312, Russia \\ E-mail: S.Loktev@gmail.com,natanzons@mail.ru \\ URL: http://www.hse.ru/en/org/persons/23004570 \\ URL: http://www.hse.ru/en/org/persons/14026884 \\ $\ddagger$ Institute of Theoretical and Experimental Physics, \\ 25 Bolshaya Cheremushkinskaya Str., Moscow 117218, Russia \\ $\S$ A.N. Belozersky Institute, Moscow State University, \\ Leninskie Gory 1, Bldg. 40, Moscow 119991, Russia
}

Received December 15, 2010, in final form July 04, 2011; Published online July 16, 2011 doi:10.3842/SIGMA.2011.070

\begin{abstract}
We show that any complex (respectively real) representation of finite group naturally generates a open-closed (respectively Klein) topological field theory over complex numbers. We relate the 1-point correlator for the projective plane in this theory with the Frobenius-Schur indicator on the representation. We relate any complex simple Klein TFT to a real division ring.
\end{abstract}

Key words: topological quantum field theory; group representation

2010 Mathematics Subject Classification: 57R56; $20 \mathrm{C} 05$

\section{Introduction}

Topological quantum field theories were introduced by Schwarz [25], Atiyah [5], Segal [26], and Witten [28]. In this paper we concentrate on open-closed and Klein topological field theories. There are several approaches how to generalize the TQFT framework for non-compact and nonorientable surfaces related to different questions of physics and geometry (see $[11,13,14,17,21$, $22,27])$. The setting we describe in this paper was proposed in [1] and developed in $[2,3,4]$. It generalizes the application of TQFT to theory of Hurwitz numbers discovered in [10].

In Section 1 we reformulate definitions of closed, open-closed, and Klein topological field theories in useful for us form (for shortness let us omit the word "quantum" there). We recall that categories of these theories are equivalent to categories of Frobenius pairs, Cardy-Frobenius algebras and equipped Cardy-Frobenius algebras respectively (this is proved in $[9,1]$ and widely generalized in $[23,24])$. Therefore constructions of a topological field theories are reduced to constructions of (equipped) Cardy-Frobenius algebras.

In Section 2 we prove that the group algebra and the center of group algebra of any finite group $G$ form a semi-simple equipped Cardy-Frobenius algebra over any number field. We call it regular. Also we construct a real equipped Cardy-Frobenius algebra from a real division ring and show that any semi-simple complex equipped Cardy-Frobenius algebra can be obtained from it by complexification. Then we present full description of Regular complex algebras of a group in these terms.

In Section 3 we prove that the center of group algebra together with the intertwining algebra of any representation of $G$ generates a Cardy-Frobenius algebra and that this algebra is equipped 
if the representation is real. For representations, that appear from group actions, we relate this construction with the one proposed in [4].

\section{Topological field theories and related algebras}

\subsection{Closed topological field theories}

The simplest variant of topological field theory is closed topological field theory $([5,9]$, see $[16]$ for more references). In this case we consider oriented closed surfaces without boundary. Also we fix a finite-dimensional vector space $A$ over a field $\mathbb{K}$ with basis $\alpha_{1}, \ldots, \alpha_{N}$ and associate a number $\left\langle a_{1}, a_{2}, \ldots, a_{n}\right\rangle_{\Omega}$ to each system of vectors $a_{1}, a_{2}, \ldots, a_{n} \in A$ situated at a set of points $p_{1}, p_{2}, \ldots, p_{n}$ on a surface $\Omega$ (Fig. 1 ).

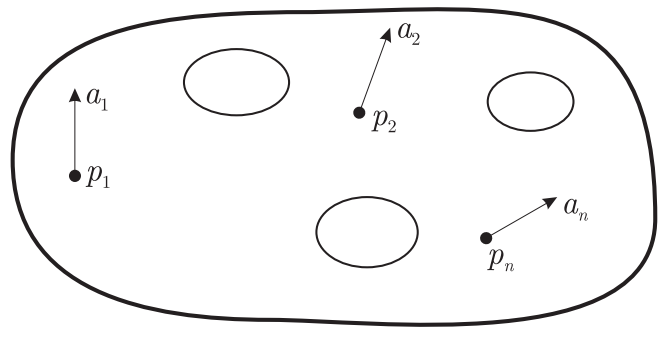

Figure 1.

We assume that the numbers $\left\langle a_{1}, a_{2}, \ldots, a_{n}\right\rangle_{\Omega}$ are invariant with respect to any homeomorphisms of surfaces with marked points. Moreover, we postulate that the system $\left\{\left\langle a_{1}, a_{2}, \ldots\right.\right.$, $\left.\left.a_{n}\right\rangle_{\Omega}\right\}$ consists of multilinear forms and satisfies a non-degeneracy axiom and cut axioms.

The non-degeneracy axiom says that the matrix $\left(\left\langle\alpha_{i}, \alpha_{j}\right\rangle_{S^{2}}\right)_{1 \leq i, j \leq N}$ is non-degenerate. By $F_{A}^{\alpha_{i}, \alpha_{j}}$ denote the inverse matrix.

The cut axioms describes evolution of $\left\langle a_{1}, a_{2}, \ldots, a_{n}\right\rangle_{\Omega}$ by cutting and collapsing along contours $\gamma \subset \Omega$. Indeed, there are two cut axioms related to different topological types of contours.

If $\gamma$ decomposes $\Omega$ into $\Omega^{\prime}$ and $\Omega^{\prime \prime}$ (Fig. 2)

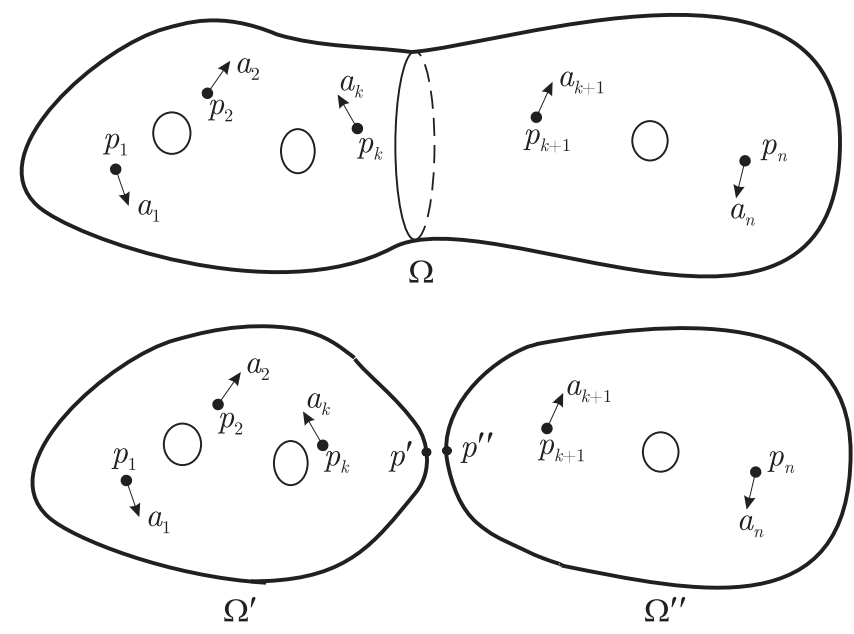

Figure 2.

then

$$
\left\langle a_{1}, a_{2}, \ldots, a_{n}\right\rangle_{\Omega}=\sum_{i, j}\left\langle a_{1}, a_{2}, \ldots, a_{k}, \alpha_{i}\right\rangle_{\Omega^{\prime}} F_{A}^{\alpha_{i}, \alpha_{j}}\left\langle\alpha_{j}, a_{k+1}, a_{k+2}, \ldots, a_{n}\right\rangle_{\Omega^{\prime \prime}} .
$$


If $\gamma$ does not decompose $\Omega$ (Fig. 3)

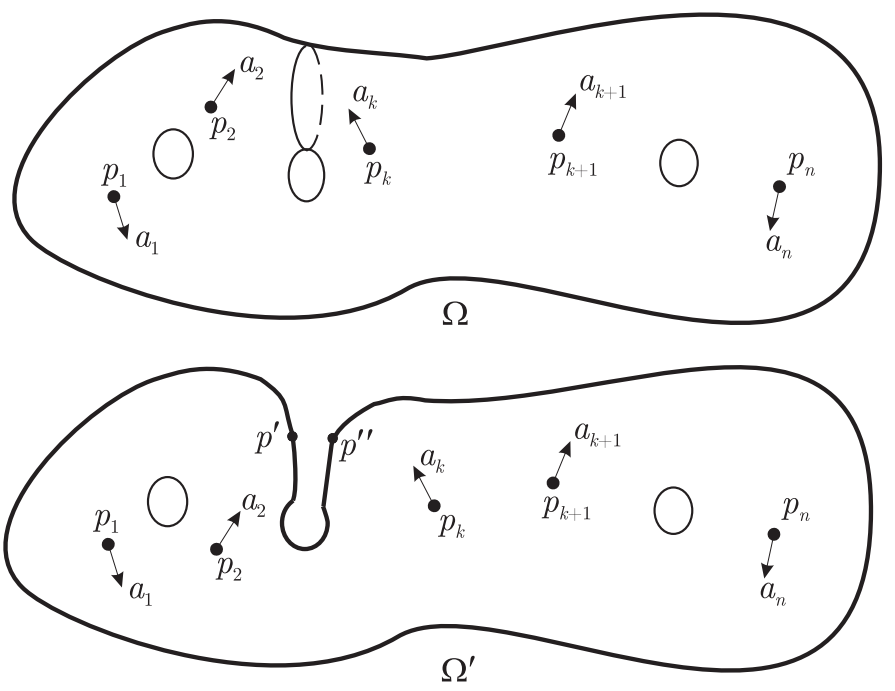

Figure 3.

then

$$
\left\langle a_{1}, a_{2}, \ldots, a_{n}\right\rangle_{\Omega}=\sum_{i, j}\left\langle a_{1}, a_{2}, \ldots, a_{n}, \alpha_{i}, \alpha_{j}\right\rangle_{\Omega^{\prime}} F_{A}^{\alpha_{i}, \alpha_{j}}
$$

The first consequence of the topological field theory axioms is a structure of algebra on $A$. Namely, the multiplication is defined by $\left\langle a_{1} a_{2}, a_{3}\right\rangle_{S^{2}}=\left\langle a_{1}, a_{2}, a_{3}\right\rangle_{S^{2}}$, so the numbers $c_{i j}^{k}=$ $\sum_{s}\left\langle\alpha_{i}, \alpha_{j}, \alpha_{s}\right\rangle_{S^{2}} F_{A}^{\alpha_{s}, \alpha_{k}}$ are structure constants for this algebra. The cut axiom gives (Fig. 4)
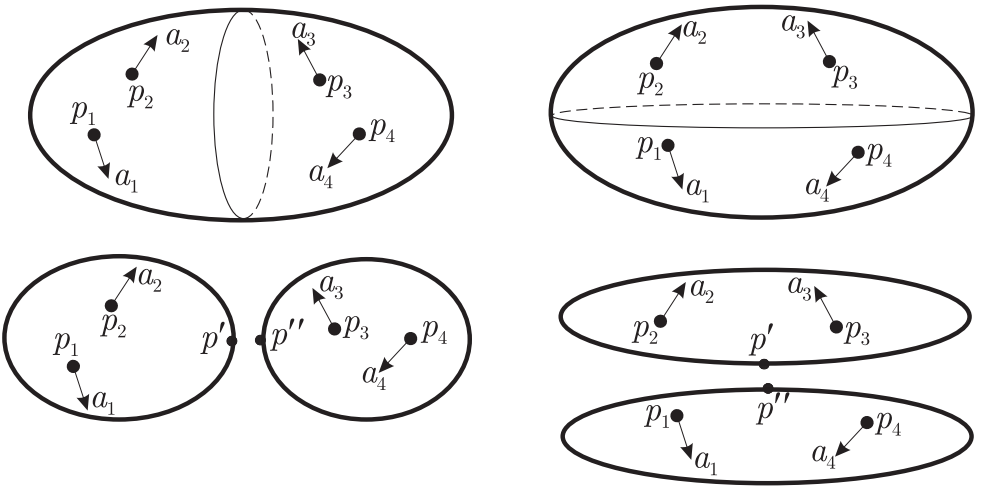

Figure 4.

$$
\begin{gathered}
\sum_{i, j}\left\langle a_{1}, a_{2}, \alpha_{i}\right\rangle_{S^{2}} F_{A}^{\alpha_{i}, \alpha_{j}}\left\langle\alpha_{j}, a_{3}, a_{4}\right\rangle_{S^{2}}=\left\langle a_{1}, a_{2}, a_{3}, a_{4}\right\rangle_{S^{2}} \\
=\sum_{i, j}\left\langle a_{2}, a_{3}, \alpha_{i}\right\rangle_{S^{2}} F_{A}^{\alpha_{i}, \alpha_{j}}\left\langle\alpha_{j}, a_{4}, a_{1}\right\rangle_{S^{2}} .
\end{gathered}
$$

Therefore $\sum_{s, t} c_{i j}^{s} c_{s k}^{t}=\sum_{s, t} c_{j k}^{s} c_{s i}^{t}$ and thus $A$ is an associative algebra. The unit of the algebra $A$ is given by the vector $\sum_{i}\left\langle\alpha_{i}\right\rangle_{S^{2}} F_{A}^{\alpha_{i}, \alpha_{j}} \alpha_{j}$. The linear form $l(a)=\langle a\rangle_{S^{2}}$ is a counit, also it defines a non-degenerate invariant bilinear form $\left(a_{1}, a_{2}\right)_{A}=l\left(a_{1} a_{2}\right)=\left\langle a_{1}, a_{2}\right\rangle_{S^{2}}$ on $A$. The topological invariance makes all marked points $p_{i}$ equivalent and, therefore, $A$ is a commutative algebra. 
Following [3] we say that $\left(A, l_{A}\right)$ is a Frobenius pair if $A$ is a symmetric Frobenius algebra [12], that is an algebra with a unit and an invariant non-degenerate symmetric bilinear form, and the bilinear form is given by $\left(a_{1}, a_{2}\right)=l_{A}\left(a_{1} \cdot a_{2}\right)$.

Thus, $\left(A, l_{A}\right)$ is a commutative Frobenius pair, moreover, the construction gives a functor $\mathcal{F}$ from the category of closed topological field theories to the category of commutative Frobenius pairs.

Theorem 1 ([9]). The functor $\mathcal{F}$ is an equivalence between categories of closed topological field theories and commutative Frobenius pairs.

The Frobenius structure gives an explicit formula for correlators:

$$
\left\langle a_{1}, a_{2}, \ldots, a_{n}\right\rangle_{\Omega}=l_{A}\left(a_{1} a_{2} \cdots a_{n}\left(K_{A}\right)^{g}\right),
$$

where $K_{A}=\sum_{i j} F_{A}^{\alpha_{i}, \alpha_{j}} \alpha_{i} \alpha_{j}$ and $g$ is genus of $\Omega$.

Note 1. This definition of closed topological field theory is equivalent to the standard one [5] as follows. Our points $p_{1}, \ldots, p_{n}$ correspond to circles in a closed 1-dimensional variety, and the surface corresponds to the cobordism of this variety with the empty set. The following definition of open-closed topological field theories is equivalent to proposed in $[18,21]$ in a similar way.

\subsection{Open-closed topological field theories}

A more complicated variant of topological field theory is open-closed topological field theory $[1,18,20,21]$. In this case we admit oriented compact surfaces $\Omega$ with boundary $\partial \Omega$ and some marked points on $\partial \Omega$. Let us denote the interior marked points and vectors as before by $p_{1}, p_{2}, \ldots, p_{n}$ and $a_{1}, a_{2}, \ldots, a_{n} \in A$. Here we introduce a special notation $q_{j}^{i}$ for the boundary marked points, where the upper index $i=1 \ldots s$ corresponds to a connected component of $\partial \Omega$ (that is a boundary contour of $\Omega$ ). The lower index $j$ is individual for any boundary contour, it counts the points consequently on the circle, following the direction determined by the orientation of $\Omega$. The vectors $b_{j}^{i}$ attached to $q_{j}^{i}$ belong to another vector space $B$ over $\mathbb{K}$ with basis $\beta_{1}, \ldots, \beta_{M}$ (Fig. 5).

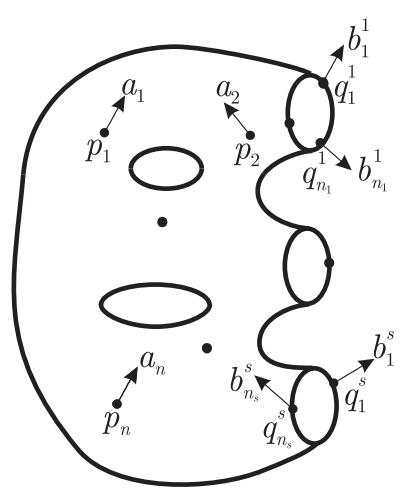

Figure 5.

Let us denote the corresponding correlator by $\left\langle a_{1}, \ldots, a_{n},\left(b_{1}^{1}, \ldots, b_{n_{1}}^{1}\right), \ldots,\left(b_{1}^{s}, \ldots, b_{n_{s}}^{s}\right)\right\rangle_{\Omega}$ to keep in mind this picture. Note that diffeomorphisms of $\Omega$ can induce any permutation of $a_{i}$, but only cyclic permutations in each group $b_{1}^{i}, \ldots, b_{n_{i}}^{i}$.

We suppose that topological invariance axiom and all axioms of closed topological field theory are fulfilled for interior marked points and cut-contours. Thus open-closed topological field theory also generates a commutative Frobenius pair $\left(A, l_{A}\right)$. Also we impose an additional non-degeneracy axiom and cut axioms related to the boundary. 
The additional non-degeneracy axiom says that for any disk $D$ with two marked boundary points the matrix $\left(\left\langle\beta_{i}, \beta_{j}\right\rangle_{D}\right)$, is non-degenerate. By $F_{B}^{\beta_{i}, \beta_{j}}$ denote the inverse matrix. It play for "segment-cuts" the same "gluing role" that $F_{A}^{\alpha_{i}, \alpha_{j}}$ for "contour-cuts".

In open-closed topological field theory we consider cuts by simple segments $[0,1] \rightarrow \Omega$ such that the image of 0 and 1 belongs to the boundary. Then there are three topological types of such cuts (Fig. 6).
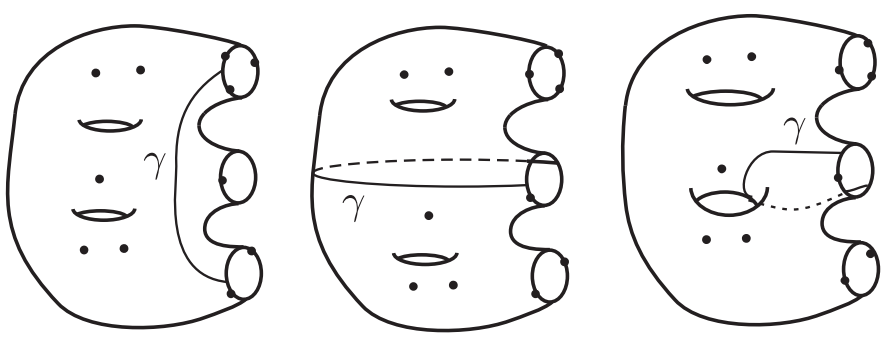

Figure 6.

Using such cuts one can reduce any marked oriented surface to elementary marked surfaces from the next list (Fig. 7). Four surfaces from the upper row are spheres and the remaining three are disks.

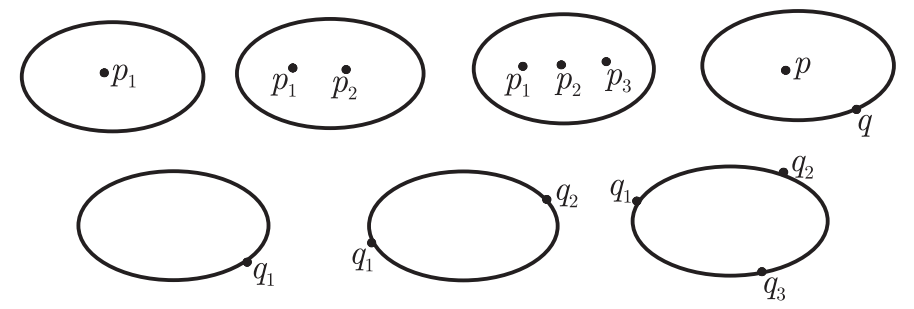

Figure 7 .

Three topological types of segments provide three new cut axioms. For example, the axiom for the cut of type 2 (Fig. 8) is
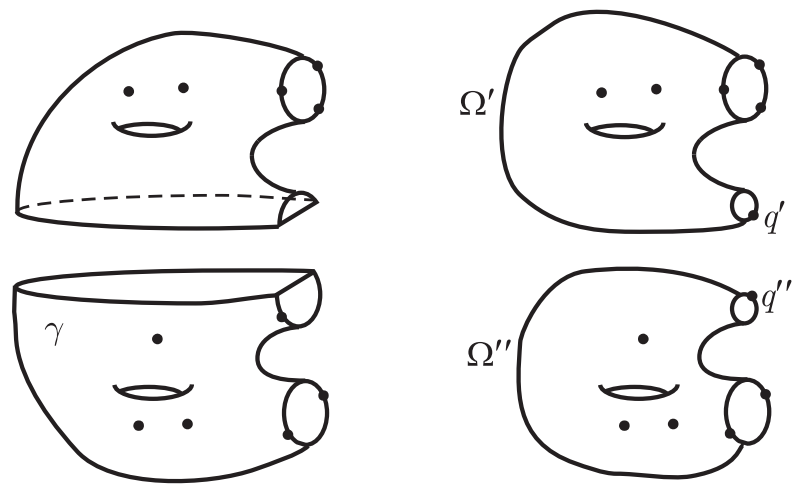

Figure 8.

$$
\begin{aligned}
& \left\langle a_{1}, a_{2}, \ldots, a_{n},\left(b_{1}^{1}, \ldots, b_{n_{1}}^{1}\right), \ldots,\left(b_{1}^{s}, \ldots, b_{n_{s}}^{s}\right)\right\rangle_{\Omega} \\
& =\sum_{i, j}\left\langle a_{1}, a_{2}, \ldots, a_{k},\left(b_{1}^{1}, \ldots, b_{n_{1}}^{1}\right), \ldots,\left(b_{1}^{t}, \ldots, b_{n_{t}^{\prime}}^{t}, \beta_{i}\right)\right\rangle_{\Omega^{\prime}} F_{B}^{\beta_{i}, \beta_{j}} \\
& \quad \times\left\langle a_{k+1}, \ldots, a_{n},\left(\beta_{j}, b_{n_{t}^{\prime}+1}^{t}, \ldots, b_{n_{t}}^{t}\right), \ldots,\left(b_{1}^{s}, \ldots, b_{n_{s}}^{s}\right)\right\rangle_{\Omega^{\prime \prime}} .
\end{aligned}
$$


The correlators for the disk $D$ with up to three boundary points $\left\langle\left(b_{1}\right)\right\rangle_{D},\left\langle\left(b_{1}, b_{2}\right)\right\rangle_{D}$ and $\left\langle\left(b_{1}, b_{2}, b_{3}\right)\right\rangle_{D}$ give us a Frobenius pair $\left(B, l_{B}\right)$ with structure constants defined in a usual way: $d_{i j}^{k}=\sum_{s}\left\langle\left(\beta_{i}, \beta_{j}, \beta_{s}\right)\right\rangle_{D} F_{B}^{\beta_{s}, \beta_{k}}$.

The associativity of $B$ follows from the picture below (Fig. 9)
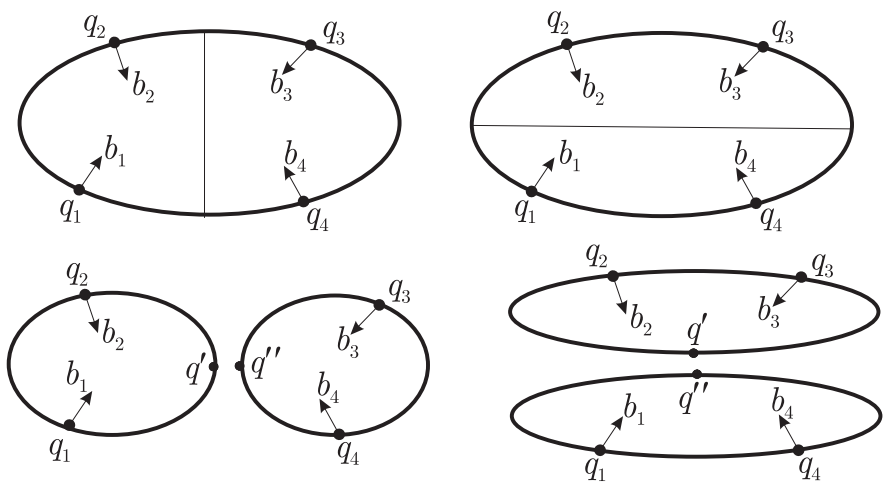

Figure 9 .

Thus $\left\langle\left(b_{1}, b_{2}, b_{3}, b_{4}\right)\right\rangle_{D}$ is equal both to $\sum_{i}\left\langle\left(b_{1}, b_{2}, \beta_{i}\right)\right\rangle_{D} F_{B}^{\beta_{i}, \beta_{j}}\left\langle\left(\beta_{j}, b_{3}, b_{4}\right)\right\rangle_{D}$ as well as to $\sum_{i}\left\langle\left(b_{2}, b_{3}, \beta_{i}\right)\right\rangle_{D} F_{B}^{\beta_{i}, \beta_{j}}\left\langle\left(\beta_{j}, b_{4}, b_{1}\right)\right\rangle_{D}$. However the algebra $B$ is not commutative in general, because there is no homeomorphisms of disk that interchanges $q_{1}$ with $q_{2}$ and preserves $q_{3}$. Nevertheless, calculating corellators for two boundary points, we have $l_{B}\left(b_{1} b_{2}\right)=l_{B}\left(b_{2} b_{1}\right)$ that makes the bilinear form on $B$ symmetric.

The correlator $\langle a,(b)\rangle_{D}: A \times B \rightarrow \mathbb{C}$ together with non-degenerate bilinear forms $\left\langle a_{1}, a_{2}\right\rangle_{S^{2}}$ : $A \times A \rightarrow \mathbb{C},\left\langle\left(b_{1}, b_{2}\right)\right\rangle_{D}: B \times B \rightarrow \mathbb{C}$ generates two homomorphisms of vector spaces $\phi: A \rightarrow B$ and the dual one $\phi^{*}: B \rightarrow A$.

Let us deduce some consequences from additional topological axioms.

Proposition 1. We have

1) $\phi$ is an algebra homomorphism,

2) $\phi(A)$ belongs to center of $B$,

3) $\left(\phi^{*}\left(b^{\prime}\right), \phi^{*}\left(b^{\prime \prime}\right)\right)_{A}=\operatorname{Tr} W_{b^{\prime} b^{\prime \prime}}^{B}$, where the operator $W_{b^{\prime} b^{\prime \prime}}^{B}: B \rightarrow B$ is $W_{b^{\prime} b^{\prime \prime}}^{B}(b)=b^{\prime} b b^{\prime \prime}$.

The last condition is known as the Cardy condition, it appeared in papers $[7,8]$.

So introduce the notion of a Cardy-Frobenius algebra $\left(\left(A, l_{A}\right),\left(B, l_{B}\right), \phi\right)$ formed by

1) a commutative Frobenius pair $\left(A, l_{A}\right)$;

2) an arbitrary Frobenius pair $\left(B, l_{B}\right)$;

3) an algebra homomorphism $\phi: A \rightarrow B$ such that $\phi(A)$ is contained in the center of $B$ and $\left(\phi^{*}\left(b^{\prime}\right), \phi^{*}\left(b^{\prime \prime}\right)\right)_{A}=\operatorname{Tr} W_{b^{\prime} b^{\prime \prime}}^{B}$

Thus, we construct a functor $\mathcal{F}$ from the category of open-closed topological field theories to the category of Cardy-Frobenius algebras $\left(\left(A, l_{A}\right),\left(B, l_{B}\right), \phi\right)$.

Note 2. This conditions are equivalent to (3.33), (3.44) in [19], where $\phi$ is called zipper and $\phi^{*}$ is called cozipper.

Theorem 2 ([1], see also [19]). The functor $\mathcal{F}$ is an equivalence between categories of open-closed topological field Theories and Cardy-Frobenius algebras. 
The structure of Cardy-Frobenius algebra provides an explicit formula for correlators:

$$
\begin{aligned}
& \left\langle a_{1}, a_{2}, \ldots, a_{n},\left(b_{1}^{1}, \ldots, b_{n_{1}}^{1}\right), \ldots,\left(b_{1}^{s}, \ldots, b_{n_{s}}^{s}\right)\right\rangle_{\Omega} \\
& \quad=l_{B}\left(\phi\left(a_{1} a_{2} \cdots a_{n} K_{A}^{g}\right) b_{1}^{1} \cdots b_{n_{1}}^{1} V_{K_{B}}\left(b_{1}^{2} \cdots b_{n_{2}}^{2}\right) \cdots V_{K_{B}}\left(b_{1}^{s} \ldots b_{n_{s}}^{s}\right)\right)
\end{aligned}
$$

where the operator $V_{K_{B}}: B \rightarrow B$ is given by $V_{K_{B}}(b)=F_{B}^{\beta_{i}, \beta_{j}} \beta_{i} b \beta_{j}$, and $g$ is genus of $\Omega$.

\subsection{Klein topological field theories}

The orientability restriction is indeed avoidable, the corresponding settings were introduced in [1] as Klein topological field theory. It is an extension of open-closed topological field theory to arbitrary compact surfaces (possible non-orientable and with boundary) equipped by a finite set of marked points with local orientation of their vicinities.

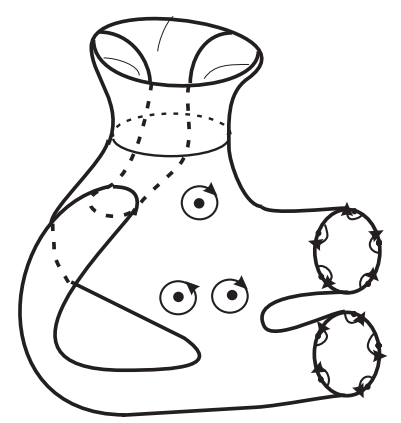

Figure 10.

In the same way as in open-closed topological field theory in order to calculate a correlator we attach vectors from a space $A$ (respectively $B$ ) to interior (resp. boundary) marked points on the surface.

We assume that topological invariance axiom and all axioms of open-closed topological field theory are fulfilled for cuts that belong to any orientable part of the surface. Thus Klein topological field theory also generates a Cardy-Frobenius algebra $\left(\left(A, l_{A}\right),\left(B, l_{B}\right), \phi\right)$.

Non-orientable surfaces gives 4 new types of cuts $(2$ types of cuts by segments and 2 types of cuts by contours) (Fig. 11).
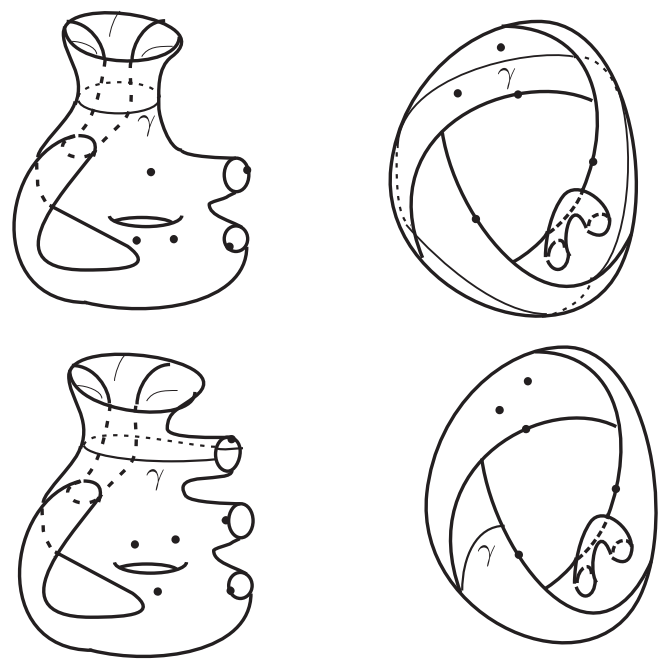

Figure 11. 
The full system of cuts provides a way to reduce any marked non-orientable surface to marked surfaces from list on Fig. 7 and the projective plane with one marked point.

Four new topological type of cuts give four new topological axioms. Three of them are similar to introduced above, and the axiom for the cut of type 2 should be different because there is only one new contour after the cut. For this case introduce an element $U \in A$ such that

$$
\begin{aligned}
& \left\langle a_{1}, a_{2}, \ldots, a_{n},\left(b_{1}^{1}, \ldots, b_{n_{1}}^{1}\right), \ldots,\left(b_{1}^{s}, \ldots, b_{n_{s}}^{s}\right)\right\rangle_{\Omega} \\
& \quad=\left\langle a_{1}, a_{2}, \ldots, a_{n}, U,\left(b_{1}^{1}, \ldots, b_{n_{1}}^{1}\right), \ldots,\left(b_{1}^{s}, \ldots, b_{n_{s}}^{s}\right)\right\rangle_{\Omega^{\prime}}
\end{aligned}
$$

Then it follows that $U$ is dual to the 1-point correlator for the projective plane, namely let $l_{U}(a)=\langle a\rangle_{\mathbb{R P}^{2}}: A \rightarrow \mathbb{K}$, then $l_{A}(U a)=l_{U}(a)$.

Now suppose that there are linear involutions $\star: A \rightarrow A$ and $\star: B \rightarrow B$, such that applying $\star$ inside the correlator gives the same answer as changing the local orientation around corresponding points. Sometimes we will write $c^{\star}=\star(c)$. Let us describe the algebraic consequences of these assumptions.

\section{Proposition 2 ([1]). We have}

1) the involution $\star: A \rightarrow A$ is automorphism, the involution $\star: B \rightarrow B$ is anti-automorphism (that is $\left.\left(b_{1} b_{2}\right)^{\star}=b_{2}^{\star} b_{1}^{\star}\right)$,

2) $l_{A}\left(x^{\star}\right)=l_{A}(x), l_{B}\left(x^{\star}\right)=l_{B}(x), \phi\left(x^{\star}\right)=\phi(x)^{\star}$,

3) $U^{2}=F_{A}^{\alpha_{i}, \alpha_{j}} \alpha_{i} \alpha_{j}^{\star}$, let us denote it by $K_{A}^{\star}$,

4) $\phi(U)=F_{B}^{\beta_{i}, \beta_{j}} \beta_{i} \beta_{j}^{\star}$, let us denote it by $K_{B}^{\star}$.

Summarizing these properties, introduce the notion of an equipped Cardy-Frobenius algebra $\left(\left(A, l_{A}\right),\left(B, l_{B}\right), \phi, U, \star\right)$ formed by

1) a Cardy-Frobenius algebra $\left(\left(A, l_{A}\right),\left(B, l_{B}\right), \phi\right)$;

2) an anti-automorphisms $\star: A \rightarrow A$ and $\star: B \rightarrow B$ such that $l_{A}\left(x^{\star}\right)=l_{A}(x), l_{B}\left(x^{\star}\right)=$ $l_{B}(x), \phi\left(x^{\star}\right)=\phi(x)^{\star}$

3) an element $U \in A$ such that $U^{2}=K_{A}^{\star}$ and $\phi(U)=K_{B}^{\star}$.

Thus, we constructed a functor $\mathcal{F}$ from the category of Klein topological field theory to the category of equipped Cardy-Frobenius algebras $\left(\left(A, l_{A}\right),\left(B, l_{B}\right), \phi, U, \star\right)$.

Theorem 3 ([1]). The functor $\mathcal{F}$ is an equivalence between categories of Klein topological field theories and equipped Cardy-Frobenius algebras.

The structure of equipped Cardy-Frobenius algebra provides an explicit formula for correlators on non-orientable surfaces:

$$
\begin{aligned}
& \left\langle a_{1}, a_{2}, \ldots, a_{n},\left(b_{1}^{1}, \ldots, b_{n_{1}}^{1}\right), \ldots,\left(b_{1}^{s}, \ldots, b_{n_{s}}^{s}\right)\right\rangle_{\Omega} \\
& \quad=l_{B}\left(\phi\left(a_{1} a_{2} \cdots a_{n} U^{2 g}\right) b_{1}^{1} \cdots b_{n_{1}}^{1} V_{K_{B}}\left(b_{1}^{2} \cdots b_{n_{2}}^{2}\right) \cdots V_{K_{B}}\left(b_{1}^{s} \ldots b_{n_{s}}^{s}\right)\right)
\end{aligned}
$$

where $g$ is geometrical genus of $\Omega$, that is, $g=a+1$, if $\Omega$ is the Klein bottle with $a$ handles and $g=a+\frac{1}{2}$, if $\Omega$ is the projective plane with $a$ handles. 


\section{Regular Cardy-Frobenius algebra of finite group}

\subsection{Construction of regular algebra}

In this section we present a construction that associates an equipped Cardy-Frobenius algebra and, therefore, a Klein topological field theory to any finite group $G$.

By $|M|$ denote cardinality of a finite set $M$. Let $\mathbb{K}$ be any field such that char $\mathbb{K}$ is not a divisor of $|G|$. By $B=\mathbb{K}[G]$ denote the group algebra. It can be defined as the algebra, formed by linear combinations of elements of $G$ with the natural multiplication, as well as the algebra of $\mathbb{K}$-valued functions on $G$ with multiplication defined by convolution. It has a natural structure of a Frobenius pair with $l_{B}(f)=f(1)$. Note that $l_{B}(f)=\operatorname{Tr}_{\mathbb{K}[G]} f /|G|$.

The center $A=Z(B)$ with the functional $l_{A}(f)=f(1) /|G|$ forms a Frobenius pair as well. Take $U=\sum_{g \in G} g^{2} \in A$.

Let $\phi$ be the natural inclusion from $A$ to $B$. Let $\star: B \rightarrow B$ be the antipode map, sending $g$ to $g^{-1}$. This map preserves the center, so we have a map $\star: A \rightarrow A$, compatible with the inclusion $\phi$.

Theorem 4. The data above form a semi-simple equipped Cardy-Frobenius algebra over $\mathbb{K}$.

Proof. First let us show that $U^{2}=K_{A}^{\star}$. For a conjugation class $\alpha \subset G$ let $E_{\alpha}=\sum_{g \in \alpha} g$. Then $E_{\alpha}$ form a basis of $A$.

Note that $\left(E_{\alpha}, E_{\alpha^{-1}}\right)_{A}=|\alpha|$ and $\left(E_{\alpha}, E_{\beta}\right)_{A}=0$ otherwise, hence as $E_{\alpha}^{\star}=E_{\alpha^{-1}}$ we have

$$
\begin{aligned}
K_{A}^{\star} & =\frac{1}{|G|} \sum_{\alpha} \frac{E_{\alpha}^{2}}{|\alpha|}=\frac{1}{|G|} \sum_{\alpha} \sum_{g, g^{\prime} \in \alpha} \frac{g g^{\prime}}{|\alpha|} \\
& =\frac{1}{|G|} \sum_{\alpha} \sum_{g \in \alpha, h \in G} \frac{g h^{-1} g h}{|\alpha|} \frac{|\alpha|}{|G|}=\sum_{g, h \in G} g h^{-1} g h=\sum_{a, b \in G} a^{2} b^{2}=U^{2},
\end{aligned}
$$

where $a=g h^{-1}, b=h$.

Also we have $K_{B}^{\star}=\sum_{x, y \in G} l_{B}\left(x y^{-1}\right) x y=\sum_{g \in G} g^{2}=U$. It remains to prove the Cardy condition $l_{A}\left(\phi^{*}(x) \phi^{*}(y)\right)=\operatorname{Tr} W_{x, y}^{B}$.

We have $\operatorname{Tr} W_{x, y}^{B}=|\{g \mid x g y=g\}|=\left|\left\{g \mid y=g^{-1} x^{-1} g\right\}\right|$. This number is zero if $x^{-1}$ and $y$ are in different conjugation classes, and it is $\frac{|G|}{|\gamma|}$ if $x^{-1}$ belongs to the conjugation class $\gamma$ of $y$. On the other hand, $\phi^{*}(y)=\sum_{g \in G} g^{-1} y g=\frac{|G|}{|\gamma|} \sum_{h \in \gamma} h=\frac{|G|}{|\gamma|} E_{\gamma}$. Thus the number $l_{A}\left(\phi^{*}(x) \phi^{*}(y)\right)$ is exactly the same.

We denote the constructed algebra by $H_{\mathbb{K}}^{G}$ and call it the regular algebra of $G$. This algebra is semi-simple due to semi-simplicity of the group algebra. Our next aim is full description of $H_{\mathbb{R}}^{G}$ and $H_{\mathbb{C}}^{G}$.

\subsection{Classification of complex semi-simple equipped Cardy-Frobenius algebras}

We call a complex Cardy-Frobenius algebra $\left(\left(A, l_{A}\right),\left(B, l_{B}\right), \phi\right)$ pseudoreal if $A=A_{R} \otimes \mathbb{C}$, $B=B_{R} \otimes \mathbb{C}$ and $\phi=\phi_{R} \otimes \mathbb{C}$ where $A_{R}, B_{R}$ are real algebras and $\phi_{R}: A_{R} \rightarrow B_{R}$ is an homomorphism. It appears (see Theorem 5 ) that any simple complex equipped Cardy-Frobenius algebra is pseudoreal.

Let $\mathbb{D}$ be a division algebra over $\mathbb{R}$, that is, $\mathbb{R}, \mathbb{C}$ or $\mathbb{H}$. For each $\mathbb{D}$ introduce a family of real semi-simple equipped Cardy-Frobenius algebras. 
Namely, let $n$ be an integer, $\mu \in \mathbb{C} \backslash 0$, put $d=\operatorname{dim}_{\mathbb{R}} \mathbb{D}$. Introduce

$$
\begin{aligned}
& B_{R}=\operatorname{Mat}_{n}(\mathbb{D}), \quad l_{B_{R}}(x)=\mu \Re e \operatorname{Tr}(x) \quad \text { for } x \in \operatorname{Mat}_{n}(\mathbb{D}), \\
& A_{R}=Z(\mathbb{D}), \quad l_{A_{R}}(a)=\mu^{2} \Re e(a) / d \quad \text { for } \quad a \in Z(\mathbb{D}), \quad \phi_{R}(a)=a \operatorname{Id} \in Z\left(B_{R}\right) .
\end{aligned}
$$

For $z \in \mathbb{D}$ by $\bar{z}$ denote the conjugated element. The involution $\star_{R}$ is defined by $a^{\star_{R}}=\bar{a}$ for $a \in A_{R}, x^{\star}=\bar{x}^{t}$ for $x \in B_{R}$, where ${ }^{t}$ means transposition of a matrix. Now take

$$
U_{R}=\frac{2-d}{\mu} \in A_{R}
$$

Denote this data $\left(\left(A_{R}, l_{A_{R}}\right),\left(B_{R}, l_{B_{R}}\right), \phi_{R}, U_{R}, \star_{R}\right)$ by $H_{n, \mu}^{\mathbb{D}}$.

Proposition 3. The object $H_{n, \mu}^{\mathbb{D}}$ is a semi-simple real equipped Cardy-Frobenius algebra.

Proof. Introduce a natural projection $Z: \mathbb{D} \rightarrow Z(\mathbb{D})$ sending $x \in \mathbb{D}$ to $x$ for $\mathbb{D}=\mathbb{R}, \mathbb{C}$ and to $\Re e(x)$ for $\mathbb{D}=\mathbb{H}$. We have $\phi_{R}^{*}(x)=Z(\operatorname{Tr}(x)) d / \mu$. On the other hand, a direct calculation in the standard basis shows that

$$
\operatorname{Tr} W_{x, y}^{B}=\operatorname{Tr} W_{\operatorname{Tr}(x), \operatorname{Tr}(y)}^{\mathbb{D}}=d \Re e(Z(\operatorname{Tr}(x)) Z(\operatorname{Tr}(y))),
$$

so $\left(\phi^{*}(x), \phi^{*}(y)\right)=\operatorname{Tr} W_{x, y}^{B}$.

Another observation is that $K_{B_{R}}^{\star}=K_{\mathbb{D}} \mathrm{Id} / \mu$, where $K_{\mathbb{D}}$ is the Casimir element of $\mathbb{D}$ with respect to the form $(a, b)=a \bar{b}$. We have $K_{\mathbb{D}}=(2-d)$, so $\phi\left(U_{R}\right)=K_{B}$. At last, $K_{A_{R}}=d / \mu^{2}$ for $A_{R}=\mathbb{R}$ and $K_{A_{R}}=0$ for $A_{R}=\mathbb{C}$, so $U_{R}^{2}=K_{A_{R}}$.

Theorem 5. Any semi-simple equipped Cardy-Frobenius algebra $\left(\left(A, l_{A}\right),\left(B, l_{B}\right), \phi, U, \star\right)$ over $\mathbb{C}$ is a direct sum of $H_{n_{i}, \mu_{i}}^{\mathbb{D}_{i}} \otimes \mathbb{C}$ and $\operatorname{Ker}(\phi)$.

Proof. We deduce the statement from a classification of equipped Cardy-Frobenius algebras performed in [1]. To identify $H_{n_{i}, \mu_{i}}^{\mathbb{D}_{i}} \otimes \mathbb{C}$ with the algebras introduced there let us describe $H_{n_{i}, \mu_{i}}^{\mathbb{D}_{i}} \otimes \mathbb{C}$ in detail.

- If $\mathbb{D}=\mathbb{R}$, then $A \cong \mathbb{C}$ with the identical involution $x^{\star}=x$ and the linear form $l_{A}(z)=\mu^{2} z$, $U=\frac{1}{\mu} \in A ; B \cong \operatorname{Mat}_{n}(n, \mathbb{C})$ equipped with the involutive anti-automorphism $\star: X \mapsto X^{t}$, and the linear form $l_{B}(X)=\mu \operatorname{Tr} X$. The homomorphism $\phi: A \rightarrow B$ sends the unit to the identity matrix;

- If $\mathbb{D}=\mathbb{C}$, then $A \cong \mathbb{C} \oplus \mathbb{C}$ with the involution $(x, y)^{\star}=(y, x)$ for $(x, y) \in \mathbb{C} \oplus \mathbb{C}$ and the linear form $l_{A}(x, y)=\mu^{2}(x+y) / 4, U=0 ; B \cong \operatorname{Mat}_{n}(n, \mathbb{C}) \oplus \operatorname{Mat}_{n}(n, \mathbb{C})$ with the linear form $l_{B}(X, Y)=\mu(\operatorname{Tr} X+\operatorname{Tr} Y) / 2$ and the involutive anti-automorphism $\star:(X, Y) \mapsto$ $\left(Y^{t}, X^{t}\right)$. The homomorphism $\phi: A \rightarrow B$ is given by the equality $\phi(x, y)=(x E, y E)$.

- If $\mathbb{D}=\mathbb{H}$, then $\left(A, l_{A}, \star\right)$ is the same as for $\mathbb{D}=\mathbb{R}$, but $U=-\frac{2}{\mu} \in A ; B \cong \operatorname{Mat}_{2 m}(\mathbb{C})$ with the linear form $l_{B}(X)=\mu \operatorname{Tr} X / 2$. A matrix $X \in B$ we can present in block form as $X=\left(\begin{array}{ll}m_{11} & m_{12} \\ m_{21} & m_{22}\end{array}\right)$. Then the involute anti-automorphism $\star: X \mapsto X^{\tau}$ is given by the formula $X^{\tau}=\left(\begin{array}{cc}m_{22}^{t} & -m_{12}^{t} \\ -m_{21}^{t} & m_{11}^{t}\end{array}\right)$, in other words, $X^{\tau}$ is the matrix adjoint to $X$ with respect to a natural symplectic form. The homomorphism $\phi: A \rightarrow B$ sends the unit to the identity matrix.

Note 3. There are real equipped Cardy-Frobenius algebras not isomorphic to $H_{n, \mu}^{\mathbb{D}}$. A simplest example is a generalization of $H_{n, \mu}^{\mathbb{R}}$ with the same Cardy-Frobenius structure, but the involution $\star$ defined as the transposition with respect to a bi-linear form with non-trivial signature. 


\subsection{Description of complex regular algebra}

Let us denote complex representations of $G$ by capital Latin letters (such as $V$ ) and real representation by Greek letters (such as $\pi$ ). Note that irreducible real representations can be split into 3 types (see [15]):

- Real type: $\operatorname{End}(\pi)=\mathbb{R}$ and $\pi \otimes_{\mathbb{R}} \mathbb{C}$ is irreducible;

- Complex type: $\operatorname{End}(\pi)=\mathbb{C}, \pi \otimes_{\mathbb{R}} \mathbb{C} \cong V^{+} \oplus V^{-}, V^{+}$is not isomorphic to $V^{-}$(but $\left.V^{+} \cong\left(V^{-}\right)^{*}\right)$

- Quaternionic type: $\operatorname{End}(\pi)=\mathbb{H}, \pi \otimes_{\mathbb{R}} \mathbb{C} \cong V^{0} \oplus V^{0}$.

Let $\operatorname{Ir}_{\mathbb{D}}(G)$ be the set of isomorphism classes of corresponding irreducible real representations.

Theorem 6. We have isomorphisms of equipped algebras

$$
\begin{aligned}
& H_{G}^{\mathbb{R}} \cong \bigoplus_{\mathbb{D}=\mathbb{R}, \mathbb{C}, \mathbb{H}} \bigoplus_{\pi \in \operatorname{Ir}_{\mathbb{D}}(G)} H_{\frac{\operatorname{dim} \pi}{\operatorname{dim} \mathbb{D}}, \frac{\operatorname{dim} \pi}{|G|}}^{\mathbb{D}}, \\
& H_{G}^{\mathbb{C}} \cong \bigoplus_{\mathbb{D}=\mathbb{R}, \mathbb{C}, \mathbb{H}} \bigoplus_{\pi \in \operatorname{Ir}_{\mathbb{D}}(G)} H_{\frac{\operatorname{dim} \pi}{\operatorname{dim} \mathbb{D}}, \frac{\operatorname{dim} \pi}{|G|}}^{\mathbb{D}} \otimes \mathbb{C} .
\end{aligned}
$$

Proof. Let us show (2), then (3) follows because $\mathbb{C}[G] \cong \mathbb{R}[G] \otimes \mathbb{C}$. By the Wedderburn theorem

$$
\mathbb{R}[G] \cong \bigoplus_{\mathbb{D}} \bigoplus_{\pi \in \operatorname{Ir}_{\mathbb{D}}(G)} \operatorname{Mat}_{\frac{\operatorname{dim} \pi}{\operatorname{dim} \mathbb{D}}}(\mathbb{D})
$$

where the map $\mathbb{R}[G] \rightarrow \operatorname{Mat}_{\frac{\operatorname{dim} \pi}{\operatorname{dim} \mathbb{D}}}(\mathbb{D})$ is the action of $\mathbb{R}[G]$ on $\pi \cong \mathbb{D}^{\frac{\operatorname{dim} \pi}{\operatorname{dim} \mathbb{D}}}$. Due to the classification theorem it is enough to identify the map $\star$ and the constant $\mu$ on each summand with the same in $H_{n, \mu}^{\mathbb{D}}$.

Concerning $\star$, choose an invariant scalar product on $\pi$. As $\pi$ is irreducible, this invariant bilinear form is unique up to a scalar. Then $\star$ is just the conjugation with respect to this form. By the orthogonalization process we can suppose that $\pi \cong \mathbb{D} e_{1} \oplus \cdots \oplus \mathbb{D} e_{m}$, where $\left\{e_{l}\right\}$ is a set of orthogonal vectors.

Note that $\operatorname{Mat}_{\frac{\operatorname{dim} \pi}{\operatorname{dim} \mathbb{D}}}(\mathbb{D})$ is the tensor product of its subalgebras $\operatorname{Mat}_{\frac{\operatorname{dim} \pi}{\operatorname{dim} \mathbb{D}}}(\mathbb{R})$ and $\mathbb{D}$. In the basis $\left\{e_{l}\right\}$ we identify the action of $\star$ on $\operatorname{Mat}_{\frac{\operatorname{dim} \pi}{\operatorname{dim} \mathbb{D}}}(\mathbb{R})$ with the matrix transposition. For $\mathbb{D}$ note that it also acts by right multiplication, an this action commutes with the action of $\mathbb{R}[G]$, hence this right action preserves the bilinear form up to a scalar. Then it follows that the set $e_{l}, i e_{l}$ $($ for $\mathbb{D} \supset \mathbb{C}), j e_{l}$ and $k e_{l}($ for $\mathbb{D}=\mathbb{H})$ form an orthogonal basis. In this basis imaginary elements of $\mathbb{D}$ act by skew-symmetric matrices, so $\star$ acts on $\mathbb{D}$ as the standard conjugation.

It remains to find $\mu$ for a summand corresponding to each irreducible real representation $\pi$. Let $e_{\pi} \in A$ be the idempotent corresponding to $\pi$. It acts on $\mathbb{R}[G]$ by projection onto the corresponding summand in (4). Then from the definition of the regular algebra we have $l_{B}\left(\phi\left(e_{\pi}\right)\right)=\operatorname{Tr}_{\mathbb{R}[G]} e_{\pi} /|G|=\frac{(\operatorname{dim} \pi)^{2}}{|G| \operatorname{dim} \mathbb{D}}$. But from the definition of $H_{n, \mu}^{\mathbb{D}}$ we have $l_{B}\left(\phi\left(e_{\pi}\right)\right)=\mu n=\mu \operatorname{dim} \pi / \operatorname{dim} \mathbb{D}$. So $\mu=\frac{\operatorname{dim} \pi}{|G|}$.

Corollary 1 (cf. [15]). Let $\pi \in \operatorname{Ir}_{\mathbb{D}}(G)$ be an irreducible real representation of $G$. Then $\operatorname{Tr}(U)$ on $\pi$ is equal to $(2-\operatorname{dim} \mathbb{D})|G|$

Proof. The element $U$ acts on $\pi \in I r_{\mathbb{D}}(G)$ by the same scalar as on $H_{\frac{\operatorname{dim} \pi}{\operatorname{dim} \mathbb{D}}, \frac{\operatorname{dim} \pi}{|G|}}^{\mathbb{D}}$. Substituting the definition (1) for $U$ and multiplying by $\operatorname{dim} \pi$, we obtain the proposed formula.

Such an element $U$ is known as Frobenius-Schur indicator (see [15]). It provides an easy way to determine type of $\pi$. It appears in a similar situation in conformal field theories (see $[6,13])$. 
Note 4. Note that Corollary 1 is applicable to a complex representation in the same way. Indeed any irreducible complex representation $V$ can be obtained as a summand in $\pi \otimes \mathbb{C}$ for a real irreducible representation $\pi$. Then the action of $U$ on $V$ also determines type of $\pi$.

\section{Cardy-Frobenius algebras of representations}

\subsection{Cardy-Frobenius algebra of a representation}

By $\mathbb{K}$ denote a field. Let $\rho$ be a finite-dimensional representation (possibly reducible) of a finite group $G$ over $\mathbb{K}$, suppose that $|G|$ is not divisible by char $\mathbb{K}$. Fix an invertible element $\mu \in$ $Z(\mathbb{K}[G])$. Set $A=Z(\mathbb{K}[G])$ with $l_{A}$ sending an element $x$ to the value of $\mu^{2} x /|G|$ at the unit element. Let $B=\operatorname{End}_{G}(\rho)$ be the algebra of intertwining operators on $\rho$ with $l_{B}(x)=$ $\operatorname{Tr}_{\rho}(\mu x /|G|)$. As the center of $\mathbb{C}[G]$ acts on $\rho$ by intertwining operators, we have a natural map $\phi: A \rightarrow B$.

Proposition 4. The data above form a semi-simple complex Cardy-Frobenius algebra. Let us denote it by $H_{\mu}^{\rho}$.

Proof. As $\mu$ is invertible, the scalar product on $A$ and $B$ defined by $l_{A}$ and $l_{B}$ are nondegenerate, so we have two Frobenius algebras.

Note that $\mathbb{K}$ can be included to an algebraically closed field $\overline{\mathbb{K}}$ and that is enough to check the Cardy condition over $\overline{\mathbb{K}}$. The algebra $\overline{\mathbb{K}}[G]$ is generated by orthogonal idempotents $\left\{e_{i}\right\}$, corresponding to irreducible complex representations $\rho_{i}$ over $\overline{\mathbb{K}}$.

The element $\mu$ acts on $\rho_{i}$ by a scalar, let us denote it by $\mu\left(\rho_{i}\right)$. Our aim is to show that the Cardy condition is equivalent to

$$
\left(e_{i}, e_{i}\right)_{A}=\mu^{2}\left(\rho_{i}\right)\left(\frac{\operatorname{dim} \rho_{i}}{|G|}\right)^{2},
$$

then we know it for the regular representation, so it follows for the general case.

If $\rho=\sum \rho_{i}^{\oplus m_{i}}$ then $B \cong \oplus_{i=1}^{s} \operatorname{Mat}_{n_{i}}(\overline{\mathbb{K}})$. Note that for $x=\sum x_{i} \in \oplus_{i} \operatorname{Mat}_{n_{i}}(\overline{\mathbb{K}})$ and a similar element $y=\sum y_{i}$ we have $\operatorname{Tr} W_{x, y}^{B}=\sum_{i} \operatorname{Tr} x_{i} \operatorname{Tr} y_{i}$.

Note that $\mu e_{i}=\mu\left(\rho_{i}\right) e_{i}$, therefore we have

$$
\phi^{*}(x)=|G| \sum_{i=1}^{s} e_{i} \frac{\operatorname{Tr} x_{i}}{\mu\left(\rho_{i}\right) \operatorname{dim} \rho_{i}},
$$

so $\left(\phi^{*}(x), \phi^{*}(y)\right)_{A}=|G|^{2} \sum_{i}\left(e_{i}, e_{i}\right)\left(\operatorname{dim} \rho_{i}\right)^{2} \operatorname{Tr} x_{i} \operatorname{Tr} y_{i}$. Comparing it with $\operatorname{Tr} W_{x, y}^{B}$ for arbitrary $x$ and $y$, we deduce the condition (5).

\subsection{Equipped Cardy-Frobenius algebra of a real representation}

Now suppose that $\mathbb{K}=\mathbb{R}$, so $\rho$ is a real representation of $G$. Then there is a non-degenerate symmetric invariant bilinear form on $\rho$. So for any operator $x \in \operatorname{End}(\rho)$ there exists a unique adjoint operator $x^{\tau} \in \operatorname{End}(\rho)$. The map sending $x$ to $x^{\tau}$ is an anti-involution of $\operatorname{End}(\rho)$, preserving the subalgebra $\operatorname{End}_{G}(\rho)$. Thus we obtain a map $\star: \operatorname{End}_{G}(\rho) \rightarrow \operatorname{End}_{G}(\rho)$.

As before, the involution on $A=\mathbb{C}[G]$ is defined by sending $g \rightarrow g^{-1}$, and $U=\sum_{g \in G} g^{2} \in A$. Also we suppose that $\mu$ is invariant with respect to this involution, that is, $\mu(g)=\mu\left(g^{-1}\right)$. Note that such $\mu$ form a vector space with dimension equal to the number of real representations of $G$. 
Theorem 7. The data above makes $H_{\mu}^{\rho}$ a semi-simple complex equipped Cardy-Frobenius algebra. Moreover, we have $\rho \cong \bigoplus_{\pi \in \operatorname{Ir}(G)} n_{\pi} \pi$ and

$$
H_{\mu}^{\rho} \otimes \mathbb{C} \cong \bigoplus_{\mathbb{D}=\mathbb{R}, \mathbb{C}, \mathbb{H}} \bigoplus_{\pi \in \operatorname{Ir}_{\mathbb{D}}(G)} H_{n_{\pi}, \mu(\pi) \frac{\mathrm{dim} \pi}{|G|}}^{\mathbb{D}} \otimes \mathbb{C}
$$

Proof. The involution $\star$ on $A$ is compatible with the involution $\star$ on $B$ because sending $g \rightarrow g^{-1}$ corresponds to the action on the dual representation, and this action can be expressed by adjoint operators with respect to an invariant bilinear form.

By Maschke's theorem we have $\rho \cong \bigoplus_{\pi \in \operatorname{Ir}(G)} n_{\pi} \pi$. Note that there is a unique up to a scalar symmetric invariant bilinear form on a real irreducible representation. Therefore, this sum can be written as an orthogonal sum such that the restriction of the form to each irreducible representation coincides with the scalar product up to a sign. Then it follows similarly to the case of regular representation that $H_{\mu}^{\rho} \otimes \mathbb{C} \cong \bigoplus H_{n_{\pi}, \mu(\pi) \frac{\operatorname{dim} \pi}{|G|}}^{\mathbb{D}} \otimes \mathbb{C}$, in particular, $\left(K_{A}^{\star}\right)^{2}=U=K_{B}^{\star}$ for $H_{\mu}^{\rho} \otimes \mathbb{C}$ and therefore for $H_{\mu}^{\rho}$ itself.

Corollary 2. Any semi-simple equipped complex Cardy-Frobenius algebra can be constructed from a real representation of a finite group.

Proof. By $Q_{8}$ denote the group of 8 elements $\pm 1, \pm i, \pm j, \pm k$ with the natural quaternion multiplication. It has one 4-dimensional quaternionic type representations over $\mathbb{R}$ and four 1dimensional real type representations. Then the group $Q_{8} \times \mathbb{Z} / 3 \mathbb{Z}$ has all the types of representations, so using Cartesian product of several copies of this group by Theorem 7 and Theorem 6 we obtain an arbitrary semi-simple equipped complex Cardy-Frobenius algebra as a summand.

\subsection{Group action case}

A particular case of this construction was already discovered in [4]. Suppose that the group $G$ acts on a finite set $X$. Let $\pi_{X}=\mathbb{R} X$ be the real representation of $G$ in the vector space formed by formal linear combinations of the elements of $X$.

Let $H_{e}^{\pi_{X}}=\left(\left(A, l_{A}\right),\left(B, l_{B}\right), \phi, U, \star\right)$. Then an explicit construction of $B$ is proposed in [4].

The group $G$ acts on $X^{n}=X \times \cdots \times X$ by formula $g\left(x_{1}, \ldots, x_{n}\right)=\left(g\left(x_{1}\right), \ldots, g\left(x_{n}\right)\right)$. Let $\mathcal{B}_{n}=X^{n} / G$. By Aut $\bar{x}$ denote the stabilizer of element $\bar{x} \in X_{n}$. Indeed for $\bar{x}=\left(x_{1}, \ldots, x_{n}\right)$ we have Aut $\bar{x}=\cap_{i}$ Aut $x_{i}$. Cardinality of this subgroup $\mid$ Aut $\bar{x} \mid$ depends only on the orbit of $\bar{x}$, so we consider it as a function on $\mathcal{B}_{n}$.

By $B_{X}$ denote the vector space generated by $\mathcal{B}_{2}$. The involution $\left(x_{1}, x_{2}\right) \mapsto\left(x_{2}, x_{1}\right)$ generates the involution $\star_{X}: B_{X} \rightarrow B_{X}$. Introduce a bi-linear and a three-linear form on $B_{X}$ as follows:

$$
\left(b_{1}, b_{2}\right)_{X}=\frac{\delta_{b_{1}, b_{2}^{\star}}}{\mid \text { Aut } b_{1} \mid}, \quad\left(b_{1}, b_{2}, b_{3}\right)_{X}=\sum_{\left(x_{1}, x_{2}\right) \in b_{1},\left(x_{2}, x_{3}\right) \in b_{2},\left(x_{3}, x_{1}\right) \in b_{3}} \frac{1}{\left|\operatorname{Aut}\left(x_{1}, x_{2}, x_{3}\right)\right|} .
$$

Define a multiplication on $B_{X}$ by $\left(b_{1} b_{2}, b_{3}\right)_{X}=\left(b_{1}, b_{2}, b_{3}\right)_{X}$. The element $e=\sum_{x \in X}(x, x)$ is a unit of $B_{X}$. At last, let $l_{B_{X}}(b)=(b, e)_{X}$.

Theorem 8. We have an isomorphism $B \cong B_{X}$ identifying $l_{B}$ with $l_{B_{X}}$ and $\star$ with $\star_{X}$.

Proof. Essentially, it was done in [4]. Elements $\left(x_{1}, x_{2}\right) \in X \times X$ enumerates matrix units $E_{x_{1}, x_{2}} \in \operatorname{End}\left(\pi_{X}\right)$, so to any orbit $b \in \mathcal{B}_{2}$ we correspond the operator $\sum_{\left(x_{1}, x_{2}\right) \in b} E_{x_{1}, x_{2}} \in$ $\operatorname{End}_{G}\left(\pi_{X}\right)$. One can check by a direct computation that this map is an algebra homomorphism and that the trace $l_{B}$ can be written as $l_{B_{X}}$. Clearly, this homomorphism is injective and, as the operator $\sum_{g \in G} g$ on $\operatorname{End}\left(\pi_{X}\right)$ is the projection to the subspace of invariants, it is surjective, so we have $B_{X} \cong B$. At last, the involution $\star_{X}$ corresponds to transposition of a matrix in the natural orthonormal basis of $\pi_{X}$, hence it corresponds to $\star$. 
Note 5. This construction defines a structure of real equipped Cardy-Frobenius algebra on the Hecke algebra $H \backslash G / H$ for an arbitrary subgroup $H \subset G$. To this end one can take $X$ to be the left coset $G / H$ with the natural action of $G$.

\section{Acknowledgements}

We are grateful to P. Deligne, B. Feigin, Yu. Manin, S. Shadrin and V. Turaev for useful discussions. Part of this work was done during the stays of S.N. at Max-Planck-Institute in Bonn, he is grateful to MPIM for their hospitality and support. The work of S.N. was partly supported by grants RFBR-11-01-00289, N.Sh-8462.2010.1 and the Russian government grant 11.G34.31.0005. The work of S.L. was partly supported by grants: N.Sh-3035.2008.2, RFBR-0901-00242, SU-HSE award No.09-09-0009, RFBR-CNRS-07-01-92214, RFBR-IND-08-01-91300, RFBR-CNRS-09-01-93106 and P. Deligne 2004 Balzan prize in mathematics.

\section{References}

[1] Alexeevski A., Natanzon S., Noncommutative two-dimensional topological field theories and Hurwitz numbers for real algebraic curves, Selecta Math. (N.S.) 12 (2006), 307-377, math.GT/0202164.

[2] Alexeevski A., Natanzon S., Algebra of Hurwitz numbers for seamed surfaces, Russian Math. Surveys 61 (2006), no. 4, 767-769.

[3] Alexeevski A., Natanzon S., Algebra of bipartite graphs and Hurwitz numbers of seamed surfaces, Izv. Math. 72 (2008), 627-646.

[4] Alexeevski A., Natanzon S., Hurwitz numbers for regular coverings of surfaces by seamed surfaces and Cardy-Frobenius algebras of finite groups, in Geometry, Topology, and Mathematical Physics, Amer. Math. Soc. Transl. Ser. 2, Vol. 224, Amer. Math. Soc., Providence, RI, 2008, 1-25, arXiv:0709.3601.

[5] Atiyah M., Topological quantum field theories, Inst. Hautes Études Sci. Publ. Math. (1988), no. 68, $175-186$.

[6] Bantay P., The Frobenius-Schur indicator in conformal field theory, Phys. Lett. B 394 (1997), 87-88, hep-th/9610192.

[7] Cardy J.L., Operator content of two-dimensional conformal invariant theories, Nuclear Phys. B 270 (1986), 186-204.

[8] Cardy J.L., Effect of boundary conditions on the operator content of two-dimensional conformally invariant theories, Nuclear Phys. B 275 (1986), 200-218.

[9] Dijkgraaf R., Geometrical approach to two-dimensional conformal field theory, Ph.D. Thesis, Utrecht, 1989.

[10] Dijkgraaf R., Mirror symmetry and elliptic curves, in The Moduli Space of Curves (Texel Island, 1994), Progr. Math., Vol. 129, Birkhäuser Boston, Boston, MA, 1995, 149-163.

[11] Dubrovin B., Geometry of 2D topological field theories, in Integrable Systems and Quantum Groups (Montecatini Terme, 1993), Lecture Notes in Math., Vol. 1620, Springer, Berlin, 1996, 120-348, hep-th/9407018.

[12] Faith C., Algebra. II. Ring theory, Grundlehren der Mathematischen Wissenschaften, no. 191, SpringerVerlag, Berlin - New York, 1976.

[13] Felder G., Fröhlich J., Fuchs J., Schweigert C., Correlation functions and boundary conditions in rational conformal field theory and three-dimensional topology, Compositio Math. 131 (2002), 189-237, hep-th/9912239.

[14] Fuchs J., Runkel I., Schweigert C., TFT construction of RCFT correlators. II. Unoriented world sheets, Nuclear Phys. B 678 (2004), 511-637, hep-th/0306164.

[15] Fulton W., Harris J., Representation theory. A first course, Graduate Texts in Mathematics, Vol. 129, Springer-Verlag, New York, 1991.

[16] Kock J., Frobenius algebras and 2D topological quantum field theories, London Mathematical Society Student Texts, Vol. 59, Cambridge University Press, Cambridge, 2004.

[17] Kong L., Runkel I., Cardy algebras and sewing constraints. I, Comm. Math. Phys. 292 (2009), 871-912, arXiv:0807.3356.

[18] Lazaroiu C.I., On the structure of open-closed topological field theory in two-dimensions, Nuclear Phys. B 603 (2001), 497-530, hep-th/0010269. 
[19] Lauda A.D., Pfeiffer H., Open-closed strings: two-dimensional extended TQFTs and Frobenius algebras, Topology Appl. 155 (2008), 623-666, math.AT/0510664.

[20] Moore G., Some comments on branes, G-flux, and K-theory, Internat. J. Modern Phys. A 16 (2001), 936-944, hep-th/0012007.

[21] Moore G., Segal G., D-branes and K-theory in 2D topological field theory, hep-th/0609042.

[22] Natanzon S.M., Exstended cohomological field theories and noncommutative Frobenius manifolds, J. Geom. Phys. 51 (2003), 387-403, math-ph/0206033.

[23] Natanzon S.M., Cyclic foam topological field theory, arXiv:0712.3557.

[24] Natanzon S.M., Brane topological field theory and Hurwitz numbers for CW-complexes, arXiv:0904.0239.

[25] Schwarz A.S., The partition function of degenerate quadratic functional and Ray-Singer invariants, Lett. Math. Phys. 2 (1977/78), 247-252.

[26] Segal G., Two-dimensional conformal field theories and modular functors, in IXth International Congress on Mathematical Physics (Swansea, 1988), Hilger, Bristol, 1989, 22-37.

[27] Turaev V., Turner P., Unoriented topological quantum field theory and link homology, Algebr. Geom. Topol. 6 (2006), 1069-1093, math.GT/0506229.

[28] Witten E., Topological quantum field theory, Comm. Math. Phys. 117 (1988), 353-386. 